\title{
Rejoinder on: Latent Markov models: a review of a general framework for the analysis of longitudinal data with covariates
}

\author{
F. Bartolucci - A. Farcomeni - F. Pennoni
}

Published online: 21 August 2014

(C) Sociedad de Estadástica e Investigación Operativa 2014

First of all, we thank all the discussants and the four referees for their contribution. They helped us to clarify some of the features of the methodology and to answer questions we had in writing the paper; they also stimulated us to consider new interesting issues. All discussants mention additional important applications, including interesting examples in areas like language processing, psychology, and finance. Their contributions strengthen our original message about the mathematical and analytical flexibility of the proposed Latent Markov (LM) framework, and especially the usefulness in many areas of application in which LM models can be seen to arise as observation-driven models (Cox 1981). Furthermore, their comments confirm that generalizations of current LM models can often be directly obtained with minor adjustments to inference.

In what follows we comment on some of the issues raised by the discussants.

Böckenhold and McShane mention a very interesting and parsimonious extension of the first-order model, which can also include non-memoryless holding times. This is potentially very interesting as in many cases persistency in latent states is stronger than

This rejoinder refers to the comments available at doi:10.1007/s11749-014-0387-1; doi:10.1007/ s11749-014-0388-0; doi:10.1007/s11749-014-0389-z; doi:10.1007/s11749-014-0390-6.

\footnotetext{
F. Bartolucci

Department of Economics, University of Perugia, Perugia, Italy e-mail: bart@stat.unipg.it
}

\author{
A. Farcomeni \\ Department of Public Health and Infectious Diseases, Sapienza, University of Rome, Rome, Italy \\ e-mail: alessio.farcomeni@uniroma1.it \\ F. Pennoni $(\bowtie)$ \\ Department of Statistics and Quantitative Methods, University of Milano-Bicocca, Milan, Italy \\ e-mail: fulvia.pennoni@unimib.it
}


the model predicts, and related to the literature on semi-Markov models and hidden semi-Markov models, which are also mentioned by Visser and Speekenbrink.

Pass, Visser, Speekenbrink, Böckenhold and McShane mention continuous-time extensions of the LM framework. This is another very interesting approach. Despite this, to our knowledge the strategies for obtaining maximum likelihood estimates still need improvement and estimates obtained through available statistical packages may be sub-optimal (i.e., the estimation algorithm can be easily trapped into a local optimum of the likelihood function). When time intervals between measurements are random and informative, we believe continuous-time LM models represent the only option. On the other hand, when measurements are irregularly spaced at either fixed or non-informative times, we often find easier to include the subjectspecific times between measurement occasions as a covariate affecting the latent distribution.

Another important issue raised by Paas and Bianconcini is that of the choice of the number of latent states, and of the possible explosion of the number of parameters when the true underlying distribution is actually continuous. We have three additional points, besides those discussed in the main paper, on this still open problem. First, a careful tuning and selection of the number of latent states is needed only when the latent distribution is the primary interest for the analysis. On the other hand, when one is using random effects mostly to remove bias arising from unobserved heterogeneity, but the main interest is in the manifest distribution, a careful calibration is not crucial. In many cases the manifest estimates will be stable, leading to the same conclusions, for $k$ above a certain value. Second, even continuous latent distributions are usually well approximated with a small number of latent states. This is testified by the goodness-offit of models with small $k$. Third, when the true underlying distribution is so complex that the actual number of states is large, also commonly used parametric random effects assumptions fail. Fortunately, in our experience this situation is not very common in applications.

As discussed by Bianconcini, temporal and cross-sectional dependence may in many applications be so complex that a single subject-time specific intercept might be restrictive. A simple example is that of repeated measurements on subjects that are also clustered (e.g., in schools). In these cases one can rely on mixed LM models (van de Pol and Langeheine 1990; Altman 2007), in which time-dependent random intercepts and item-specific (time-constant) random intercepts are simultaneously included in the model; see also Bartolucci et al. (2011). In our experience, these methods are effective but may become cumbersome when a large number of random effects are used, leaving space for improvement of the inferential procedures.

Finally, we would like to thank Visser and Speekenbrink for pointing out the connections with the hidden Markov model and for the Miller (1952) reference. Some features of interest on this topic have also been developed in the Ph.D. thesis by Pennoni recently published as Pennoni (2014).

Acknowledgments F. Bartolucci and F. Pennoni acknowledge the financial support from the grant "Finite mixture and latent variable models for causal inference and analysis of socio-economic data" (FIRB-Futuro in ricerca) funded by the Italian Government (RBFR12SHVV). 


\section{References}

Altman RM (2007) Mixed hidden Markov models: an extension of the hidden Markov model to the longitudinal data setting. J Am Stat Assoc 102:201-210

Bartolucci F, Pennoni F, Vittadini G (2011) Assessment of school performance through a multilevel latent Markov Rasch model. J Educ Behav Stat 36:491-522

Cox DR (1981) Statistical analysis of time series: some recent developments. Scand J Stat 8:93-115

Miller GA (1952) Finite Markov processes in psychology. Psychometrika 17:149-167

Pennoni F (2014) Issues on the estimation of latent variable and latent class models. Scholars'Press, Saarbücken

van de Pol F, Langeheine R (1990) Mixed Markov latent class models. Sociol Methodol 20:213-247 